\title{
A Transformation Definition Metamodel for Model Transformation ${ }^{+}$
}

\author{
Jin Liu, Keqing He, Bing Li, Chengwan He, Peng Liang \\ The State Key Laboratory of Software Engineering, Wuhan University, Wuhan, China, 430072 \\ jliu@sklse.org
}

\begin{abstract}
Automated model transformation from PIMs to PSMs is a pivotal challenge of model driven development [1-3]. Since models are usually represented in a graphic manner of class diagram(CD), current graphictransformation based approach seems to be a natural approach. To overcome deficiencies of this approach, i.e. insufficiency of direct support for $\mathrm{CD}$ and explicit formal definition, we define a transformation definition metamodel, which employs the rule template match technique. The rule iteration algorithm and the rule schedule algorithm form the computing model. We also define and implement a transformation rule set for the transformation from UML class models to EJB models. Explicitly formal definition, transformation metamodel and demonstration make our work comprehensible and applicable. Besides MDD, our work also has potential to be applied in software evolution and integration.
\end{abstract}

Keywords: model transformation; metamodel

\section{Introduction}

What makes Model Driven Architecture (MDA) different from current software development approachs is model transformations, i.e. the process of automated converting one model to another model of the same system[1-3]. The major challenge for this approach is the automated model transformation from technique platform independent models (PIM) to platform specific models (PSM) [4-5], which is mainly due to the use of graphically $\mathrm{OO}$ modeling languages, e.g. UML class diagram (CD)[6]. Until now, there is no well-established foundation for transforming PIMs into PSMs[3,4,5,7].

Several approaches have been proposed to specify the technique for modelling PIM-to-PSM transformation $[3,4,8,9]$. But it is still hard to say which of them could be the viable solutions. According to the summarization in $[3,9]$, direct manipulation approaches such as Rational XDE, i.e. directly manipulating the exposed APIs, lack

+ This research was supported by NSFC(China) under grant No.60373086; Open Foundation of SKLSE under grant No.03-03; the Provincial Natural Science Foundation of Hubei under grant No.2002ABB037; Hubei Key Project under grant No. 902130819. high-level abstractions for specifying transformations. Relational approach with text style is a declarative approach which specifies the mathematical relations between elements in the source and target models [10,11]. But it may be difficult to implement such complex mathematical relationship $[3,4,9]$.

Also with a declarative manner, graph-transformation based approach different from relational approach[9]. It seems to be a natrual solution to model transformation because current OMG graphical language such as MOF and UML provide a well-established foundation for defining PIMs and PSMs[1]. Elements of graphic patterns can be expressed in the concrete syntax of their respective source or target languages familiar to developers working with a given modeling language. Examples of this approach are PROGRES and GReAT $[9,11]$. But neither of them provide direct support for CD, nor explicitly formal definition and metamodel, which makes it hard to understand and apply them $[3,4,9]$.

Practical graph-transformation-based approach should build on strict definition to guarantee automatic transformation. Thus we define a formal transformaton definition metamodel. The rule iteration and schedule algorithms form the computing model of transformation. To estimate the competency of our work, we take Kleppe and Warmer's work as a typical scenario and define the transformation rule set from CD to EJB model[12].

\section{Transformation Definition Metamodel}

To clarify model transformation, we define a model transformation $\mathcal{M} \mathcal{D}$ herein as a 7-tuple:

$$
\begin{aligned}
& \left(m m_{\text {source }}, m m_{\text {target }}, m_{\text {source }}, m_{\text {target }}, m t_{\mathcal{B T D}}, R_{S \mathcal{T}}, t_{\text {TE }}\right) \\
& \text { The source metamodel } m m_{\text {source }} \text { and the target }
\end{aligned}
$$


transformation rule set $R_{S T}$ from $m_{\text {source }}$ to $m_{\text {target }}$. Then transformation execution $t_{\mathcal{T E}}$ can execute model transformation and transform $m_{\text {source into }} m_{\text {target }}$ by implementing $R_{S \mathcal{T}}$. Understanding how $m t_{B \mathcal{B T}}$ and $t_{\text {TE }}$ interact with each other can help realize practical transformation. To this end, we define a transformation definition metamodel TDM.

To begin with, we define $m t_{\text {BTD }}$ based on UML CD. $\mathrm{CD}$ provides a modeling structure set that can be employed in most object oriented modeling activates. For the space limitation, formal definitions of basic transformation structures are not included in this paper and can be found on[14]. Following the basic CD structures, we define the structures for the rule template match technique(RTM), which prescribes how to transform source model fragments into target model fragments according to a rule. A rule is consisted of a source template rtemp $_{\text {source }}$ and a target template rtemp $p_{\text {target }}$. As a rule template match instance, a source model fragment can match rtemp $_{\text {source }}$ and produce a target model fragment according to rtemp $p_{\text {target }}$ in the same rule. Complete definitions for TMM can be found on[14]. Typical definition with model fragment transformation $m f t$ may like this:

Definition 1: Let a model fragment transformation $m f t$ be a tuple $\left(r t m i_{\text {source }}, r_{k}\right)$ that transform a source model fragment specified by $r$ tmi $i_{\text {source }}$ into a target model fragment $m f_{\text {target }}$ via transformation rule $r_{k}$, $m f t: R T M I S_{m_{\text {source }}} \times R S_{m_{\text {source }}, m_{\text {targ } e t}} \rightarrow M F_{m m_{\text {target }}}$ i.e., $m f t\left(r t m i_{\text {source }}, r_{k}\right) \mapsto m f_{\text {target }}$, where

$$
\begin{aligned}
& \quad-r_{k} \in R S_{m m_{\text {source }}, m m_{t} \text { arg } e t} \\
& -\left(m m_{\text {source }}=r_{k}\left|r t e m p_{\text {source }}\right| m m\right) \wedge\left(m m_{\text {target }}=\right. \\
& \left.\quad r_{k} \mid r \text { temp } p_{\text {target }} \mid m m\right) \wedge\left(\exists m_{\text {source }} \in M_{m m_{\text {source }}}\right) \wedge \\
& \quad\left(m_{\text {target }} \in M_{m m_{\text {target }}}\right) \\
& - \text { for a given } r \text { rtmi } i_{\text {source }} \in R T M I S_{m_{\text {source }}:} \\
& \quad m f_{\text {source }}=r t m i_{\text {source }} \mid m f \rightarrow \exists r_{k} \in R S_{m_{\text {source }}, m m_{t} \text { arget }}, \\
& \quad \exists r \text { tmi }_{\text {target }} \in R T M I S_{m_{\text {target }}}:\left(\quad r \text { rmi }_{\text {source }} \mid r t e m p=\right.
\end{aligned}
$$

$r_{k} \mid$ rtemp $\left._{\text {source }}\right) \wedge\left(r_{k} \mid\right.$ rtemp $_{\text {target }}=$

$$
\left.r m_{\text {target }} \mid r t e m p\right) \wedge\left(m f_{\text {target }}=r t m_{\text {target }} \mid m f\right) \text {. }
$$

With these basic transformation structures, we define the rule iteration algorithm and the rule schedule algorithm, which form computing model for our approach.

Rule Iteration Algorithm: A rule Iteration execution $r$ Exe $\left(m, r, m f_{\text {target_init }}\right)$ is a mapping:

$$
\begin{aligned}
& R E x e: M_{m m_{\text {source }}} \times R S_{m m_{\text {source }}, m m_{\text {targ } e t}} \rightarrow M F_{m m_{\text {target }}}, \\
& \text { i.e., }, r E x e\left(m, r, m f_{\text {target_init }}\right) \mapsto m f_{\text {target }|r t m i|} \text {, where } \\
& -m \in M_{m m_{\text {source }}} \\
& -r \in R S_{m m_{\text {source }}, m m_{\text {target }}} \\
& -m f_{\text {target },|r t m|} \in M F_{m m_{\text {target }}} \\
& -\left(r t m i=m f t \mid r t m i_{\text {source }}\right) \wedge\left(m f t\left|r t m i_{\text {source }}\right| r t e m p=\right. \\
& \left.\quad r \mid r t e m p_{\text {source }}\right)
\end{aligned}
$$

$|r t m i|$ is the number of times a rule template match instances rtmi that continuously occurs in this mapping, i.e. the number of times the rule $r$ instance executes. This process forms a sequence of a rule template match instances. Generating $m f_{\text {target },|r t m|}$ is an iterative process of implementing rule $r$.

Let $m f_{\text {target }, 0}=m f_{\text {target_init }}$;

For (i $=1$ to $|r t m i|)$

$m f_{\text {target }, i}=m f_{\text {target }, i-1} \circ_{\text {union }} m f t(r t m i, r)$

Rule Schedule Algorithm: A model transformation $M T\left(m_{\text {source }}, R S_{\text {schedule }}\right)$ is a mapping:

$M T: M_{m m_{\text {source }}} \times R S_{m m_{\text {source }}, m m_{\text {targ } e t}} \rightarrow M F_{m m_{\text {target }}}$, i.e.

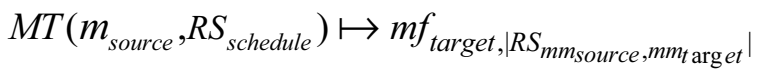

$-R S_{m m_{\text {source }}, m m_{\text {target }}}$ is the rule set of model translation from $M F_{m m_{\text {source }}}$ to $M F_{m m_{\text {target }}}$

$-\left|R S_{m m_{\text {Source }}, m m_{\text {target }}}\right|$ is the size of $R S_{m m_{\text {Source }}, m m_{t \text { arg } e t}}$.

-a rule schedule $R S_{\text {schedule }}$ is a rule execution sequence

$<r_{1}, \ldots, r_{i}, . ., r_{R S\left|m m_{\text {source }}, m m_{t} \arg e t\right|}>$ 


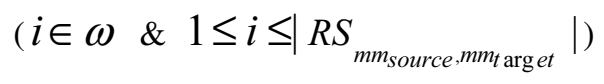

Let $m f_{\text {target }, 0}=(\varnothing, \varnothing)$

For $\left(\mathrm{i}=1\right.$ to $\left.\left|R S_{\mathrm{mm}_{\text {source }}, \mathrm{mm}_{\text {target }}}\right|\right)$

$m f_{\text {target, } \mathrm{i}}=r \operatorname{Exe}\left(m, R S_{\text {schedule }} \mid r_{\mathrm{i}-1}, m f_{\text {target } \mathrm{i}-1-1}\right)$

The process of model transformation is composed of a rule execution sequence over a source model. Each rule in $R S_{m m_{\text {source }}, m m_{\text {target }}}$ is executed based on the prior transformation output. $R S_{\text {schedule }}$ specifies the rule execution sequence. The incremental process as a whole specifies the computing model for model transformation.

A metamodels is a model which precisely defines the abstract syntax and static semantics of a particular domain-specific modeling language[13,15]. To manifest our work, we define a transformation metamodel TDM. TDM specifies the modeling concepts and their relationships that would be used to define model transformation. Modeling concepts are not only the actual domain concepts, but also standard modeling abstractions. This is especially important in the case of graphical modeling languages[13]. Instead of adding meaning to the precise syntactic and static semantic modeling specifications, implementation details usually obscure the specification and make metamodel interpretation more difficult. Metamodeling is the process of modeling metamodel.

In this paper, there are seven tasks to carry out metamodeling for our transformation metamodel:

1) Identification of the techniques in the method

Techniques types $=\{$ Class diagram, Rule template match $\}$

2) Identification of object types

Modeling concept types $=\{$ Class, Package, model fragment, model, rule template, rule, rule template match instance, model fragment transformation \}

3) Determination of properties for each object type

Properties for object types $=\{$

$<$ Class, \{Class identifier, Class name, Class stereotype, Attributes, Operations $\}>$,

$<$ Package, \{Package identifier, Package name, Package stereotype $\}>$,

$<$ model fragment, \{Class set, Class association set, Package set $\}>$,

$<$ model, $\{$ model fragment $\}>$,

$<$ rule template, $\{$ model fragment variable $\}$,

$<$ rule, \{source rule template, target rule template\}>,

$<$ rule templage match instance, \{model fragment, rule template $>$,

$<$ model fragment transformaiton, \{rule, rule template match instance $\}>$

\}

Derived property types $=\{$

<Attributes, $\{<$ Attribute, $\{$ Attribute Identifier,
Attribute Name, Attribute type $\}>\}>$,

$<$ Operations, \{<Operation, \{Operation Identifier, Operation Name, Operation type $\}>\}>$,

$<$ Class name, \{Class name identidier, Class name type $>$,

$<$ class stereotype, \{class stereotype identifier, class stereotype type $\}>$,

$<$ package name, \{package identifier, package type \}>, $<$ package stereotype, \{package stereotype identifier, \} package stereotype type $\}>$

4) Determination of relationships

Relationship
Inheritance $\}$ type $=\{$ Association, Aggregation,

5) Allocation of properties to relationship types

Properties for relationship $=\{$

$<$ Association, \{Association identifier, Association name, Association stereotype, Associaton Type, Associaiton ends \}>

\}

Derived properties for relationship $=\{$

$<$ Association ends, $\{<$ Associaion end, $\{$ role identifier, role name, class, multiplicity, association type, isNavigable $\}>\}>$

\}

6) Determination of metamodels for individual techniques

Class Diagram $=\{$

$<$ Dependency, $\{<$ Contain, $\{$ Package $\}>$,

$<$ Within, $\{$ Class set $\}>\}>$

$<$ Dependency, $\{<$ Contain, $\{$ Package $\}>$,

$<$ Within, $\{$ Class association set $\}>\}>$,

$<$ Inheritance, $\{<$ Specialization, $\{$ Class $\}>$,

$<$ Generalization, $\{$ Class $\}>\}>$,

$<$ Aggregation, $\{<$ Whole, $\{$ Class $\}>$, $<$ Part, $\{$ Class $\}>\}>$

$<$ Association, $\{<$ Associations, $\{$ Class $\}>$, $<$ Associations, $\{$ Class $\}>\}>$

\}

Rule template $=\{$

$<$ Composition, \{<Contain, \{rule template match instance $>, \quad<$ Component, $\quad$ model fragment $\}>\}\}>$,

$<$ Composition, $\{<$ Contain, $\quad$ model fragment transformaiton $\}>, \quad<$ Component, $\{$ rule template match instance $\}>\}\}>$,

$<$ Composition, $\{<$ Contain, $\{$ model fragment transformaiton $\}>,<$ Component, $\{$ rule $\}>\}\}>$,

$<$ Composition, $\{<$ Contain, $\{$ rule $\}>,<$ Component, $\{$ rule template $\}>\}\}>\quad\}$

7) Determination of linkages between separate techque

Techque linkage $=\{<$ Class diagram, rule template match $\rangle$, $\{<$ Contain, \{rule template, rule template match instance $\}>,<$ Component, $\{$ model fragment $\}>\}\}$

Synthesizing the analytical results, we complete the transformation definition metamodel (TDM) and present it in Figure 1. With this metamodel, we define transformation rule set $R_{S \mathcal{T}}$ and execute the process of transformation. 


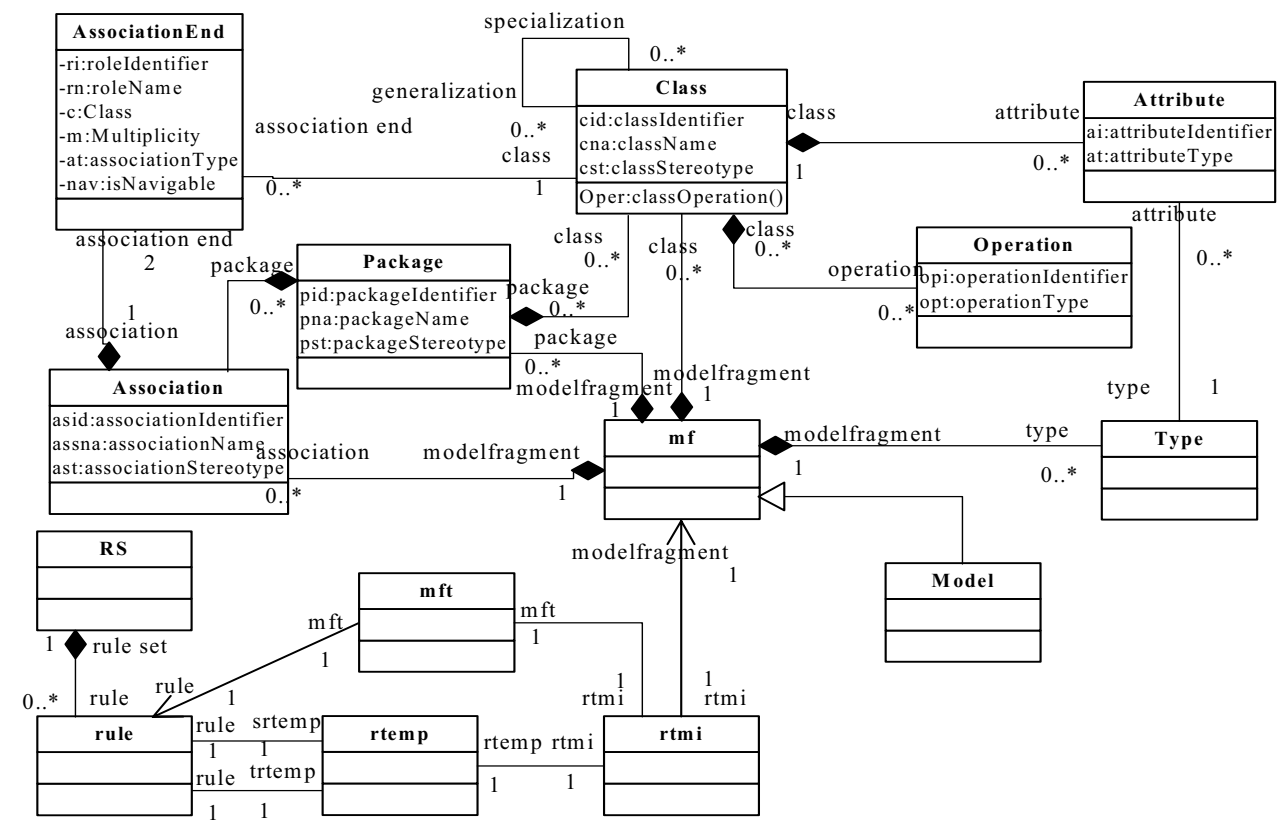

\section{Transformation Rule Set}

To estimate our work convincing, we take A. Kleppe and J. Warmer's work as our typical application scenario [12]. We define the rule set of model transformation from UML to EJB model, i.e. $\mathbb{R}_{\mathrm{UMLCD}}$ EJB according to TDM. The UML class metamodel and EJB model also contribute to the definition of $\mathcal{R}_{\mathrm{UMLCD} \cdot E J B}$ because they explicitly specify model elements and relationship between a source data model and a target data model.

EJBDataSchema is a set of classes, attributes, associations, which is treated as a EJB Component in data access. We take EJBDataSchema as a package with "EJBDataSchema" as its stereotype. EJBDataClass is a class and also a component of EJBDataSchema. EJBKeyClass is a class that is used to distinguish EJB data objects. For a class A, the outermost composition of A is a class that directly or indirectly comprises class A.

According to the analysis of these two metamodels, [12]summarizes several principles to define transformation rules:

(1)For each PIM class, transformation produces a EJBKeyClass.

(2)For each PIM class that is not a component of other classes, transformation produces an EJBEntityComponent and an EJBDataSchema.

(3)Each PIM class is transformed into an EJBDataClass that is included in an EJBDataSchema. And the EJBDataSchema is transformed from the outmost composition class of this PIM class.

(4)Each PIM association is transformed into a EJB efinition metamodel

association that is included in a EJBDataSchema. This EJBDataSchema is transformed from the outmost composition class of this PIM association.

(5)Each PIM association class is transformed into two EJBAssociation and an EJBDataClass. These EJBAssociation and EJBDataClass are included in a EJBDataSchema. This EJBDataSchema is transformed from a PIM class that is the outmost composition class. PIM class can be traced from PIM association.

(6)Each attribute is transformed into an EJB attribute of an EJBDataClass.

(7)Each operation is transformed into an EJB operation of an EJBComponent. The EJBComponent is transformed from a class that is the outmost composition class that owns this PIM operation.

In definitions of these rules, a set of any attributes is denoted by notation "anyAttribute". Similarly, a set of any operations is denoted by notation "anyOperation". OCL(Object Constrain Language) is also introduced in the definition our model transformation rules [16]. As a textual language, OCL is widely adopted in UML family and is adept at describing concise restriction information. In our work, we take advantage of OCL to enhance the strict definition of our transformation rules.

Based on these principles, we define the rule set from the UML class diagram to EJB model. Other transformation rules can be found in the appendix of this paper. Figure 2 depicts a typical rule with inheritance relationship between two classes. These rules as a whole constitute our transformation rule set. 




Figure 2. A typical transformation rule

For the sake of limited space, these transformation can not all be listed here and can be found on [14]. We take $r_{9}\left(\right.$ rtemp $_{\text {source }}$, rtemp $\left.p_{\text {target }}\right)$ as a typical rule definition which specifies a model transformation from a model fragment of the source model and produces a corresponding model fragment of target model. This rule takes into account the semantics of inheritance relationship between two classes. For the specialization class in the source model, attributes and operations inherited from the generalization class are manifestly produced in target classes that are produced according to the preceding transformation principles. OCL code is attached to class of a source model fragment to restrict the occurrence condition of a rule template match.

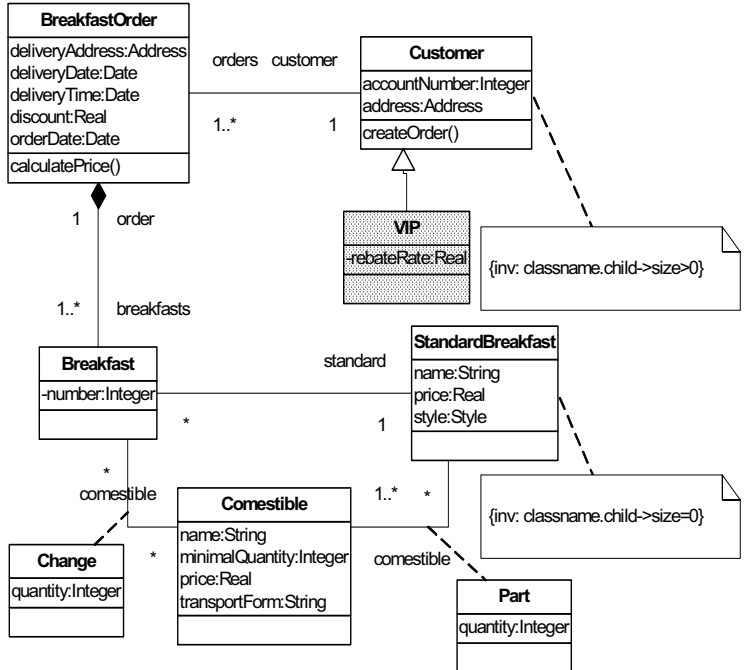

Figure 3. The PIM of breakfast order system

\section{Implementation}

We implement our rule set $\boldsymbol{R}_{\mathrm{UMLCD} \cdot E J B}$ in a transformation demonstration in [12] which is an EJB based Web Service for breakfast order. EJB model functions as the middle layer that encapsulates the business logic. Figure 3 depicts the PIM of this demonstration, which involves no details of specific implementation technique.

The process of a model transformation is consisted of

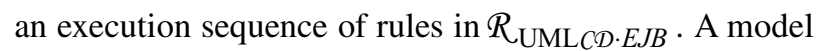
fragment transformation $m f t$ specifies the relation between a model fragment of the source model and a model fragment of the target model via a certain rule. Besides that, there are three vital aspects in the process of a rule execution, i.e., rule template match $\mathrm{rtm}_{\text {source }}$ on source model fragment $m f_{\text {source }}$, generating corresponding target model fragment $m f_{\text {target }}$ according to rule template match $\mathrm{rtm}_{\text {target }}$, and uniting the newly generated target model fragment $m f_{\text {target }}$ with the formerly generated target model fragment. Figure 4 depicts such a rule iterative execution of transformation rule instance $r E_{x} e_{i}$.

On the top of Figure 4, $r_{9}$ (rtemp $_{\text {source }}$, rtemp target $)$ is the rule in a rule iteration $r$ Exe $_{i}$ above the corresponding source model fragments and target model.

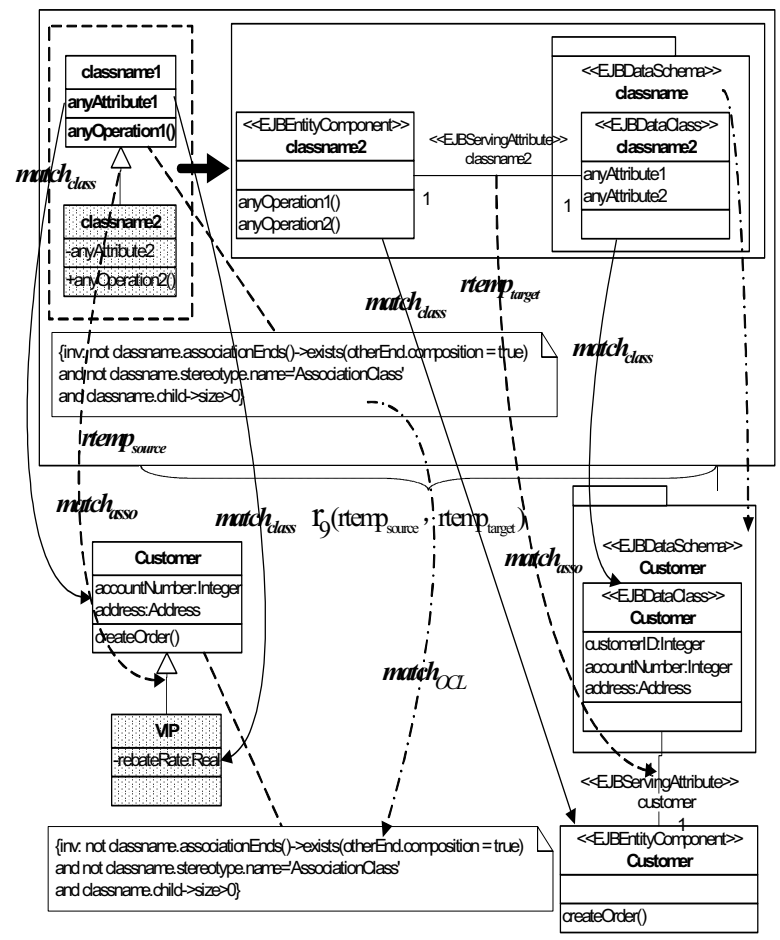

Figure 4. The rule iteration $r E x e_{i}$

On the left hand, there is an occurrence of the rule template match $\mathrm{rtm}_{\text {source }}$. Tagged with match $_{\text {class }}$, match $_{\text {asso }}$ or match $_{\text {asso }}$, lines that link elements of rtemp $p_{\text {source }}$ and model fragment denote such match. On 
the right hand, a target model fragment is accordingly generated via model fragment transformation $m f t$ ( $\left.r t m_{\text {source }}, r\right)$, which are roughly denoted with rule template match links.

The rule iteration algorithm indicates that generating target model fragment $m f_{\text {target }, \text { rtmi } \mid}$ is an iterative process of implementing rule $r_{i}$, where $|r t m i|$ is the number of rule template match instance rtmi that occur in $r$ Exe $_{i}$, and satisfies $m f_{\text {target }, i}=m f_{\text {target }, i-1}{ }^{\circ}$ union $m f t$ $(r t m, r)$. In rule instance $r E x e_{i},\left(m f_{\text {target }, 1}\right.$ $\left.=(\varnothing, \varnothing) \circ_{\text {union }} m f t(r t m, r)_{1}\right) \wedge\left(m f_{\text {target }, 2}=m f_{\text {target }, 1}\right.$ $\left.\circ_{\text {union }} m f t(r t m, r)_{2}\right) \wedge\left(m f_{\text {target },|r t m|}=m f_{\text {target }, 2}\right)$.

For the model transformation $M T$, target model fragment produced by each rule in $R S_{\text {schedule }}$ sequence has to union with the prior model fragment generated in the last transformation. But a rule instance $r E x e_{i}$ may be consisted of an execution sequence of $m f t$ s that comply with the same rule $r_{i}$. Figure 5 describes the model transformation algorithm $M T\left(m_{\text {source }}, R S_{\text {schedule }}\right)$, which realize the process of transformation execution $t_{\text {TE }}$. Due to space limitation, the target model that has been produced as the result of model transformation can be found on [14].

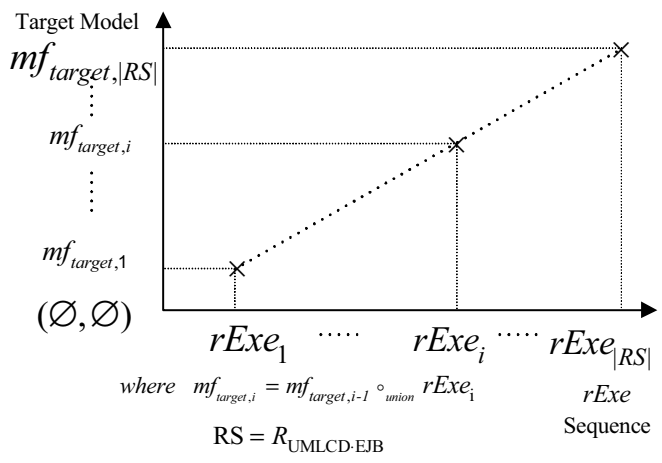

Figure 5. The process of $M T\left(m_{\text {source }}, R S_{\text {schedule }}\right)$

\section{Conclusion and Future Work}

Compared with manual modeling activities, automatic PIM-PSM model transformation encapsulates modeling patterns freqently being applied in software modeling, which improves productivity in software development. Explicitly formal definition and transformation metamodel and demonstration make our work comprehensible and applicable.Besides MDD, our work also has potential to be applied in software evolution and integration.

The original descrption of an application as an important specification renders the intention how we think of it. By analyzing current approaches in model transformation [3,9], we find that most of them only touch with syntax transformation rather than original intention transformation. We are analyzing the relationship between intention transformation and syntax transformation. Specifically, we are seeking to know whether intention transformation can be improved when the domain of applications to be built has been prescribed. Thus the transformation mechanism will be built based on the source metamodel, target metamodel and the domain ontology of models. What we have done involves syntax transformation and establishes a good foundation for our further research.

\section{References}

[1]OMG MDA Guide Ver 1.0.1, www.omg.org

[2]D. S. Frankel. Model Driven Architecture: Applying MDA to Enterprise Computing. John Wiley \& Sons, 2003

[3]S. Sendall, W. Kozaczynski, Model Transformation-the Heart and Soul of Model-Driven Software Development, IEEE Software, 20(5), 2003, pp.42-45.

[4]T. Gardner, et al. A review of OMG MOF 2.0 Query/View/Transformations, Proc of 1st International Workshop, York, UK, Nov 2003, pp178-197.

[5]A. Gerber, M. Lawley, K. Raymond, J. Steel, A. Wood, Transformation: The Missing Link of MDA, ICGT02, proceedings. LNCS vol. 2505, Springer-Verlag, 2002,pp. 90- 105 [6]OMG Unified Modeling Language 1.4, 2002, www.omg.org

[7]G. Caplat, J. L. Sourrouille, Model Mapping in MDA, Workshop in Software Model Engineering, 2002.

[8]T. Gardner, Mapping from UML to the Business Mapping from UML to BPEL4WS, OMG MDA Implementers' Workshop, Orlando, May 2003.

[9]K. Czarnecki, S. Helsen, Classification of Model Transformation Approaches, OOPSLA'03 Workshop on Generative Techniques in the Context of Model-Driven Architecture, 2003.

[10]D. H. Akehurst, S.Kent. A, Relational Approach to Defining Transformations in a Metamodel. The Unified Modeling Language 5th International Conference, Proceedings, LNCS 2460, 2002, pp243-258

[11]J. H. Hausmann, Metamodeling Relations-Relating metamodels, Model Mapping in MDA, Proc of 1st International Workshop, York, UK, Nov 2003, pp147-161.

[12]A. Kleppe, J. Warmer, W. Bast, MDA Explained: The Model Driven Architecture: Practice and Promise, Wesley, 2003

[13]D. Harel and B. Rumpe, Modeling Languages: Syntax, Semantics and All That Stuff, Part I: The Basic Stuff,Aug,2000.

[14]J. Liu and K. He. Basic structure for Transformation Definition Metamodel, SKLSE Technique Report, June, 2004. www.sklse.org/group/uml/SEFG/jliu/TechniqueReport04_06.pdf [15]Jin Liu, Keqing He, A MOP Based Constructive Reflective State Metamodel, Wuhan University Journal of Natural Sciences, 9(2), Mar, 2004, 161-166.

[16]J. Warmer, A. Kleppe, The Object Constraint Language, Getting your Models Ready for MDA, 2003, Addison-Wesley. 